(C) 2003 International Press

Adv. Theor. Math. Phys. 7 (2003) 381-404

\title{
D-branes, $B$ fields, and Ext groups
}

\author{
Andrei Căldăraru ${ }^{1}$, Sheldon Katz ${ }^{2,3}$ and Eric Sharpe ${ }^{2}$ \\ ${ }^{1}$ Department of Mathematics, University of Pennsylvania \\ David Rittenhouse Lab., 209 South 33rd Street \\ Philadelphia, PA 19104-6395 \\ ${ }^{2}$ Department of Mathematics, University of Illinois \\ 1409 W. Green St., MC-382 ,Urbana, IL 61801 \\ ${ }^{3}$ Department of Physics \\ University of Illinois at Urbana-Champaign, Urbana, IL 61801 \\ andreic@math.upenn.edu,katz@math.uiuc.edu, ersharpe@uiuc.edu
}

\begin{abstract}
In this paper we extend previous work on calculating massless boundary Ramond sector spectra of open strings to include cases with nonzero flat $B$ fields. In such cases, D-branes are no longer well-modeled precisely by sheaves, but rather they are replaced by 'twisted' sheaves, reflecting the fact that gauge transformations of the $B$ field act as affine translations of the Chan-Paton factors. As in previous work, we find that the massless boundary Ramond sector states are counted by Ext groups - this time, Ext groups of twisted sheaves. As before, the computation of BRST cohomology relies on physically realizing some spectral sequences. Subtleties that cropped up in previous work also appear here.
\end{abstract}

e-print archive: http://lanl.arXiv.org/abs/hep-th/0302099

ILL-(TH)-03-01 


\section{Contents}

1 Introduction

382

2 Overview of twisted sheaves

384

3 Parallel coincident branes on $S \hookrightarrow X$

390

4 Example

392

5 Parallel branes on submanifolds of different dimension

6 General intersecting branes

394

7 Conclusions

395

8 Acknowledgments

396

A Proofs of spectral sequences

References

403

\section{Introduction}

One of the predictions of current proposals for the physical significance of derived categories (see $[1,2,3,4]$ for an incomplete list of early references) is that massless boundary Ramond sector states of open strings connecting D-branes wrapped on complex submanifolds of Calabi-Yau's, with holomorphic gauge bundles, should be counted by mathematical objects known as Ext groups. Specifically, if we have D-branes wrapped on the complex submanifolds $i: S \hookrightarrow X$ and $j: T \hookrightarrow X$ of a Calabi-Yau $X$, with holomorphic gauge fields in $^{1} \mathcal{E} \otimes \sqrt{K_{S}^{\vee}}, \mathcal{F} \otimes \sqrt{K_{T}^{\nabla}}$, respectively, then massless boundary

\footnotetext{
${ }^{1}$ Because of the Freed-Witten anomaly, gauge fields on D-brane worldvolumes do not couple to honest bundles, but rather to twisted bundles, which is reflected in the $\sqrt{K_{S}^{v}}$ factors. A detailed explanation of the sheaf-brane correspondence, taking into account the
} 
Ramond sector states should be in one-to-one correspondence with elements of either

$$
\operatorname{Ext}_{X}^{*}\left(i_{*} \mathcal{E}, j_{*} \mathcal{F}\right)
$$

or

$$
\operatorname{Ext}_{X}^{*}\left(j_{*} \mathcal{F}, i_{*} \mathcal{E}\right)
$$

(depending upon the open string orientation).

In [5] we explicitly checked this proposal for large-radius Calabi-Yau's, by using standard well-known BCFT methods to compute the massless Ramond sector spectrum of open strings connecting such D-branes, and then relating that spectrum to Ext groups. Naively the massless Ramond sector states are not counted by Ext groups, but rather sheaf cohomology groups that are merely related to Ext groups via spectral sequences. However, a closer examination of the physics revealed that, at least in cases we understand well, those spectral sequences are realized directly in BRST cohomology, and so the massless Ramond sector spectrum is counted directly by Ext groups.

In [6] we extended the work in [5] to consider D-branes in orbifolds. We again found that massless boundary Ramond sector states are counted by Ext groups, this time on quotient stacks. Previously, open string spectra in orbifolds had only been computed for topologically trivial brane configurations, so we were able to implicitly solve the old technical challenge of calculating spectra for more general configurations. We also commented on the physical meaningfulness of quotient stacks. For example, fractional branes cannot be described as sheaves on quotient spaces - they can only be described as sheaves on quotient stacks.

Both of the papers $[5,6]$ implicitly assumed that there was no $B$ field background. In this paper we shall extend the methods of [5] to the case of nonzero, nontrivial, flat $B$ fields.

In the presence of a nontrivial $B$ field, one does not have an honest bundle on D-brane worldvolumes, essentially because in open strings gauge transformations of the $B$ field mix with transformations of the Chan-Paton factors, as

$$
\begin{aligned}
B & \mapsto B+d \Lambda \\
A & \mapsto A-\Lambda .
\end{aligned}
$$

Instead, the bundle is twisted, in the sense that the transition functions $g_{\alpha \beta}$

Freed-Witten anomaly, appears in [5]. 
no longer close to the identity on triple overlaps, but rather obey

$$
g_{i j} g_{j k} g_{k i}=\alpha_{i j k}
$$

for some $U(1)$-valued 2-cocycle $\alpha$ partly defining the $B$ field. (For a readable description of nontrivial $B$ fields, see [7]). This twisting has played an important role in, for example, the derivation $[8,9]$ of Douglas's description of discrete torsion for D-branes [10], and has also been discussed in [11, 12].

One can make rigorous mathematical sense of such twisted bundles [13], as well as analogous twisted notions of sheaf cohomology and Ext groups, as we shall review.

In this paper, after discussing how nontrivial flat $B$ fields can be described by certain elements of the group $H^{2}\left(X, \mathcal{O}_{X}^{*}\right)$, we shall compute the massless boundary Ramond sector spectrum of open strings connecting D-branes, in the presence of a $B$ field defined by $\omega \in H^{2}\left(X, \mathcal{O}_{X}^{*}\right)$, and we shall show that such states are in one-to-one correspondence with elements of twisted Ext groups, either

$$
\operatorname{Ext}_{X, \omega}^{*}\left(i_{*} \mathcal{E}, j_{*} \mathcal{F}\right)
$$

or

$$
\operatorname{Ext}_{X, \omega}^{*}\left(j_{*} \mathcal{F}, i_{*} \mathcal{E}\right)
$$

(depending upon the open string orientation).

We begin in section 2 with an overview of bundles and sheaves twisted by the $B$ field, and relevant bits of physics. In section 3 we discuss massless boundary Ramond sector spectra for open strings between parallel coincident branes. The analysis proceeds very much as in $[5,6]$, and we find that said spectra are ultimately counted by Ext groups between twisted sheaves. In section 4 we discuss an example. Unfortunately, due to a lack of known Calabi-Yau manifolds with the relevant properties, we are not able to discuss a wide variety of examples, but we do at least illustrate how the relevant calculations are performed. In section 5 we discuss massless boundary Ramond sector spectra for parallel branes on submanifolds of different dimension. In section 6 we discuss massless boundary Ramond sector spectra for general branes. Finally, in appendix A we prove some spectral sequences used in this paper.

\section{Overview of twisted sheaves}

As mentioned in the introduction, in the presence of a nontrivial $B$ field, one no longer has an honest bundle on the worldvolume of a D-brane. Ordinarily 
on triple overlaps the transition functions $g_{i j}$ of a bundle obey the condition

$$
g_{i j} g_{j k} g_{k i}=1
$$

However, $B$ field gauge transformations combine nontrivially with ChanPaton translations, as

$$
\begin{aligned}
B & \mapsto B-d \Lambda \\
A & \mapsto A+\Lambda
\end{aligned}
$$

so if the $B$ field has nonzero transition functions, then the connection on our 'bundle' picks up affine translations between coordinate charts. The effect is that the transition functions $g_{i j}$ of the 'bundle' obey the condition [14]

$$
g_{i j} g_{j k} g_{k i}=\alpha_{i j k}
$$

for a 2-cocycle $\left(\alpha_{i j k}\right)$ representing a cohomology class in $H^{2}\left(X, C^{\infty}(U(1))\right)$. These cohomology classes define gerbes, and the $\left\{g_{i j}\right\}$ satisfying (1) define twisted bundles. From the exact sequence

$$
0 \rightarrow \mathbf{Z} \rightarrow C^{\infty}(\mathbf{R}) \rightarrow C^{\infty}(U(1)) \rightarrow 0
$$

we get a map $H^{2}\left(X, C^{\infty}(U(1))\right) \rightarrow H^{3}(X, \mathbf{Z})$ which takes $[\alpha]$ to a class in $H^{3}(X, \mathbf{Z})$, the characteristic class of the gerbe. Note that $H^{2}\left(X, C^{\infty}(U(1))\right) \cong$ $H^{3}(X, \mathbf{Z})$. See [7] for more details.

Note that modulo scalars, (1) becomes

$$
\bar{g}_{i j} \bar{g}_{j k} \bar{g}_{k i}=1
$$

where $\bar{g}$ is the PGL $(n)$ image of $g \in \mathrm{GL}(n)$. If the twisted bundle has rank $r$, then (2) says that we get a cohomology class $[\bar{g}] \in H^{1}\left(X, C^{\infty}(\operatorname{PGL}(r))\right)$. From the exact sequence

$$
0 \rightarrow \mathbf{Z}_{r} \rightarrow C^{\infty}(\mathrm{GL}(r)) \rightarrow C^{\infty}(\operatorname{PGL}(r)) \rightarrow 0
$$

we get a coboundary map $H^{1}\left(X, C^{\infty}(\operatorname{PGL}(r))\right) \rightarrow H^{2}\left(X, \mathbf{Z}_{r}\right)$, taking $[\bar{g}]$ to an element of $H^{2}\left(X, \mathbf{Z}_{r}\right)$. It is straightforward to check that the image of this class in $H^{2}\left(X, C^{\infty}(U(1))\right)$ induced by the inclusion $\mathbf{Z}_{r} \rightarrow U(1)$ is precisely the class of the gerbe defined by the original $\alpha$. Since $H^{2}\left(X, \mathbf{Z}_{r}\right)$ is torsion, we see that rank $r$ twisted bundles can only exist if the class of the underlying gerbe is $r$ torsion. In particular, if the gerbe is not a torsion class, then there are no finite-rank twisted bundles at all.

Such twisted bundles are understood in mathematics (see for example [13]). One can define not only twisted bundles, but also twisted coherent 
sheaves on a space. In fact, sheaves can also be defined in terms of local data and transition functions obeying a cocycle condition (see for example [15, cor. I-14]), so we can define twisted sheaves in exactly the same form as twisted bundles. On the other hand, although twisted bundles are immediately realized in D-brane physics, the physics of twisted sheaves is more obscure. Part of what we are doing in this paper amounts to giving concrete evidence that D-branes in flat $B$ field backgrounds can be accurately modeled by twisted sheaves (see also [16] for previous work in this vein).

In this holomorphic context, the twisting is defined by an element $\alpha \in$ $H^{2}\left(X, \mathcal{O}_{X}^{*}\right)$ associated to the $B$ field. Moreover, one can also define Ext groups between twisted sheaves. If $\mathcal{S}, \mathcal{T}$ are two coherent sheaves on $X$, both twisted by the same element $\omega \in H^{2}\left(X, \mathcal{O}_{X}^{*}\right)$, then one can define $\operatorname{Ext}_{X, \omega}^{*}(\mathcal{S}, \mathcal{T})$. We will say more about such Ext groups and how to arrive at elements of $H^{2}\left(X, \mathcal{O}_{X}^{*}\right)$ from a $B$ field momentarily. First we will review twisted sheaves in more detail.

For convenience of the reader, we give the complete definition of a twisted sheaf. If $\alpha \in H^{2}\left(X, \mathcal{O}_{X}^{*}\right)$ is represented by a Cech 2-cocycle $\left(\alpha_{i j k}\right)$ on an open cover $\left\{U_{i}\right\}_{i \in I}$, with the $\alpha_{i j k}$ holomorphic, an $\alpha$-twisted sheaf $\mathcal{F}$ consists of a pair

$$
\left(\left\{\mathcal{F}_{i}\right\}_{i \in I},\left\{\phi_{i j}\right\}_{i, j \in I}\right),
$$

where $\mathcal{F}_{i}$ is a sheaf on $U_{i}$ for all $i \in I$ and

$$
\phi_{i j}:\left.\left.\mathcal{F}_{j}\right|_{U_{i} \cap U_{j}} \rightarrow \mathcal{F}_{i}\right|_{U_{i} \cap U_{j}}
$$

is an isomorphism for all $i, j \in I$, subject to the conditions:

1. $\phi_{i i}=\mathrm{Id}$;

2. $\phi_{i j}=\phi_{j i}^{-1}$

3. $\phi_{i j} \circ \phi_{j k} \circ \phi_{k i}=\alpha_{i j k} \cdot \mathrm{Id}$.

The class of twisted sheaves together with the obvious notion of homomorphism is an abelian category, the category of $\alpha$-twisted sheaves, leading to the stated notion of Ext groups by general homological algebra.

This notation is consistent, since one can prove that this category is independent of the choice of the covering $\left\{U_{i}\right\}([13,1.2 .3])$ or of the particular cocycle $\left\{\alpha_{i j k}\right\}([13,1.2 .8])$

Now a $B$-field is a connection on a gerbe. To say it is a connection means that we can express it locally as a 2 -form $B_{i}$, subject to gauge transformations $B_{i}-B_{j}=d \Lambda_{i j}$ for some 1 -forms $\Lambda_{i j}$. The field strength $H=d B$ is 
then globally defined. Since $\Lambda_{i j}+\Lambda_{j k}+\Lambda_{k i}$ is exact, we can locally write $\Lambda_{i j}+\Lambda_{j k}+\Lambda_{k i}=-i \alpha_{i j k}^{-1} d \alpha_{i j k}$, where $\alpha_{i j k}$ are local $U(1)$-valued functions, once again representing the class of the underlying gerbe. In this context, $[H] \in H^{3}(X, \mathbf{R})$ is the characteristic class of the gerbe (it actually lies in $\left.H^{3}(X, \mathbf{Z})\right)$. If in addition the $B$ field is flat, i.e. if $H=0$, then the above considerations lead to the holonomy class of $B$ in $H^{2}(X, \mathbf{R} / \mathbf{Z})$. See [7] for more details. In particular the contribution of the $B$-field to the action can be specified topologically for a closed string worldsheet.

A connection on a twisted $U(N)$ bundle in the presence of a $B$-field is analogous to the usual notion of a connection. We have a local collection of 1 forms $A_{i}$ satisfying

$$
A_{i}-\left(f_{i j}\right) A_{j}\left(f_{i j}\right)^{-1}=f_{i j}^{-1} d f_{i j}-I \Lambda_{i j}
$$

Here, $\Lambda_{i j}$ is the gauge transformation of the $B$-field as discussed above, and $I$ is the $N \times N$ identity matrix. Note that $\operatorname{Tr} F$ is not gauge invariant, but $\operatorname{Tr} F+B$ is gauge invariant.

For completeness, we describe the relationship between elements of $H^{2}\left(X, \mathcal{O}_{X}^{*}\right)$ and gerbes, at least in the case of a Calabi-Yau threefold. Extensions are straightforward. There is an exact sequence

$$
H^{2}\left(X, \mathcal{O}_{X}\right) \longrightarrow H^{2}\left(X, \mathcal{O}_{X}^{*}\right) \longrightarrow H^{3}(X, \mathbf{Z}) \longrightarrow H^{3}\left(X, \mathcal{O}_{X}\right)
$$

and on a Calabi-Yau threefold, $H^{2}\left(X, \mathcal{O}_{X}\right)=0$. Thus $H^{2}\left(X, \mathcal{O}_{X}^{*}\right)$ is the kernel of

$$
H^{3}(X, \mathbf{Z}) \rightarrow H^{3}\left(X, \mathcal{O}_{X}\right) \simeq H^{0,3}(X) .
$$

This map can be described by first mapping $H^{3}(X, \mathbf{Z}) \rightarrow H^{3}(X, \mathbf{R})$, then following with the Hodge-theoretic projection of a de Rham third cohomology class onto its $(0,3)$ component. Since an element of $H^{3}(X, \mathbf{Z})$ is real, the $(3,0)$ component vanishes whenever its $(0,3)$ component vanishes. Thus we see that given an element of $H^{3}(X, \mathbf{Z}) \cap\left(H^{2,1}(X) \oplus H^{1,2}(X)\right){ }^{2}$ we can uniquely reconstruct a corresponding element of $H^{2}\left(X, \mathcal{O}_{X}^{*}\right)$. Note that $H^{3}(X, \mathbf{Z}) \cap\left(H^{2,1}(X) \oplus H^{1,2}(X)\right)$ plays an important role in the generalized Hodge conjecture in dimension 3 . For more details on this circle of ideas, see [17].

Define the intermediate Jacobian

$$
J(X)=\left(H^{3,0}(X) \oplus H^{2,1}(X)\right)^{*} /\left(H^{3}(X, \mathbf{Z})\right),
$$

\footnotetext{
${ }^{2}$ We are not being quite precise in notation here, as $H^{3}(X, \mathbf{Z})$ does not embed in $H^{3}(X, \mathbf{R})$ in the presence of torsion.
} 
a complex torus of dimension $h^{2,1}+1$. Given a family of holomorphic curves on the Calabi-Yau $X$, parametrized by $S$, there is an Abel-Jacobi mapping

$$
\operatorname{Alb}(S)=\left(H^{1,0}(S)\right)^{*} / H_{1}(S) \rightarrow J(X) .
$$

Now $J(X)$ is not an abelian variety, but it contains an abelian variety $A(X)$ whose dimension is equal to the rank of $H^{3}(X, \mathbf{Z}) \cap\left(H^{2,1}(X) \oplus H^{1,2}(X)\right)$. In fact, $A(X)$ is the maximal abelian variety contained in $J(X)$ and orthogonal to $H^{3,0}(X)$. The generalized Hodge conjecture asserts that the Abel-Jacobi mapping is surjective for some family of curves.

For example, on the Fermat quintic threefold, $H^{3}(X, \mathbf{Z}) \cap\left(H^{2,1}(X) \oplus\right.$ $\left.H^{1,2}(X)\right)$ has rank 200 and consequently $A(X)$ is an abelian variety of dimension 200. In this case, it is straightforward to check that the Abel-Jacobi image for the family of lines on $X$ is precisely $A(X)$. Furthermore, $A(X)$ decomposes as a direct sum of genus 2 Jacobians. This genus 2 Jacobian has appeared in the physics literature in [18] and the relevant mathematics has been discussed more recently in [19].

In this paper, we are only interested in flat $B$ fields, which means that the curvature (the de Rham image of the characteristic class) vanishes, so the corresponding element of $H^{3}(X, \mathbf{Z})$ is purely torsion. When this happens, its image in $H^{3}\left(X, \mathcal{O}_{X}\right)$ necessarily vanishes for the above reasons.

In summary, the torsion subgroup of $H^{2}\left(X, \mathcal{O}_{X}^{*}\right)$ is isomorphic to the torsion subgroup of $H^{3}(X, \mathbf{Z})$. So the elements of $H^{2}\left(X, \mathcal{O}_{X}^{*}\right)$ relevant to our study of D-branes correspond to purely topological information.

As we have seen above, one can define holomorphic locally free twisted sheaves of finite rank only when the twisting is by an element of $H^{2}\left(X, \mathcal{O}_{X}^{*}\right)$ corresponding to a torsion element of $H^{3}(X, \mathbf{Z})$. The elements arising this way form the Brauer group. (A widely-believed conjecture of Grothendieck says that the elements of the Brauer group are the same as the torsion elements of $H^{2}\left(X, \mathcal{O}_{X}^{*}\right)$.) For twistings by non-torsion elements, no locally free twisted sheaves of finite rank exist. So our physical constraint of flat $B$ fields translates directly into mathematics.

In passing, we will note that there is a closely analogous phenomenon in the study of WZW models. There, one has strings propagating on a group manifold with a nontrivial $B$ field background, in which the curvature $H$ of the $B$ field is nonzero (this is the point of the Wess-Zumino term, after all). D-branes cannot wrap the entire group manifold. Instead, D-branes can only wrap submanifolds $S$ such that the restriction of the characteristic class to $S$ is torsion $[20,21]$, mirroring the statement that finite-rank locallyfree twisted sheaves only exist when the characteristic class of the twisting 
is torsion.

Another way to think about twisted holomorphic bundles is that they are associated to projective space bundles that cannot be obtained by projectivizing an ordinary holomorphic vector bundle. In detail, given a projective space bundle, imagine lifting the fibers locally from $\mathbf{P}^{n}$ to $\mathbf{C}^{n+1}$. The original projective space bundle was a bundle, so its transition functions close on triple overlaps. However, if we lift those same transition functions from $\mathbf{P}^{n}$ to $\mathbf{C}^{n+1}$, then they need only close up to the action of $\mathbf{C}^{\times}$on triple overlaps. In other words, given a projective space bundle, it need not lift to an honest vector bundle, but in general will only lift to a twisted vector bundle, twisted by an element of $H^{2}\left(X, \mathcal{O}_{X}^{*}\right)$ defined by the failure of the transition functions to close on triple overlaps.

Given two twisted sheaves $\mathcal{E}, \mathcal{F}$, one can define a twisted sheaf of local homomorphisms $\underline{\operatorname{Hom}}(\mathcal{E}, \mathcal{F})$. If $\mathcal{E}$ is twisted by $\omega \in H^{2}\left(X, \mathcal{O}_{X}^{*}\right)$ and $\mathcal{F}$ is twisted by $\omega^{\prime} \in H^{2}\left(X, \mathcal{O}_{X}^{*}\right)$, then $\underline{\operatorname{Hom}}(\mathcal{E}, \mathcal{F})$ is a twisted sheaf, twisted by $\omega^{-1} \cdot \omega^{\prime} \in H^{2}\left(X, \mathcal{O}_{X}^{*}\right)$.

In particular, if two bundles $\mathcal{E}, \mathcal{F}$ are twisted by the same element of $H^{2}\left(X, \mathcal{O}_{X}^{*}\right)$, then $\underline{\operatorname{Hom}}(\mathcal{E}, \mathcal{F})$ is an ordinary, untwisted sheaf.

We can use that fact to set up homological algebra in the usual form. If $\mathcal{S}$, $\mathcal{T}$ are two twisted sheaves, but twisted by the same element of $H^{2}\left(X, \mathcal{O}_{X}^{*}\right)$, then we can define local $\underline{\operatorname{Ext}}_{\mathcal{O}_{X}}^{n}(\mathcal{S}, \mathcal{T})$ and global $\operatorname{Ext}_{X}^{n}(\mathcal{S}, \mathcal{T})$.

This last fact is directly relevant for this paper, as in any given theory there is only a single $B$ field that twists the D-brane bundles. Thus, open string spectra are always computed between twisted sheaves that have been twisted by the same element of $H^{2}\left(X, \mathcal{O}_{X}^{*}\right)$, which means that for all our string spectrum calculations, there exists a relevant notion of Ext groups in mathematics, and the associated Ext sheaves are ordinary sheaves.

Serre duality functions for twisted sheaves much as for ordinary untwisted sheaves. For $\omega \in H^{2}\left(X, \mathcal{O}_{X}^{*}\right)$ an element of the Brauer group (i.e. corresponding to a flat $B$ field), for any two $\omega$-twisted coherent sheaves $\mathcal{S}, \mathcal{T}$ on a Calabi-Yau manifold $X$, we have

$$
\operatorname{Ext}_{X, \omega}^{i}(\mathcal{S}, \mathcal{T}) \cong \operatorname{Ext}_{X, \omega}^{n-i}(\mathcal{T}, \mathcal{S})^{*}
$$

We have spoken a great deal so far about twisted sheaves in general terms. Although our remarks apply in principle, in practice we unfortunately do not know of any examples of smooth Calabi-Yau manifolds with non-zero torsion part of $H^{2}\left(X, \mathcal{O}_{X}^{*}\right)$. 
We do know of some spaces that are close but not quite Calabi-Yau, and for the purposes of illustrating our methods with some concrete examples, we will mention a few such examples.

Our example of a space with nonzero torsion in $H^{2}\left(X, \mathcal{O}_{X}^{*}\right)$ is taken directly from the appendix of [22]. Let $W \subset \mathbf{P}^{3} \times \mathbf{P}^{3}$ be a generic hypersurface of bidegree $(2,2)$, and let $K \subset \mathbf{P}^{3}$ be the discriminant locus of the fibration $p_{1}: W \rightarrow \mathbf{P}^{3}$, where $p_{1}$ is the projection onto the first factor. One can show that $K$ is an octic surface with 80 ordinary nodes. Let $d: X \rightarrow \mathbf{P}^{3}$ be the double cover of $\mathbf{P}^{3}$ branched over $K$, and let $\pi: \tilde{X} \rightarrow X$ be the blowup of the 80 nodes of $X$, so that $\tilde{X}$ is smooth. Since we have blown-up the nodes, rather than small-resolved them, this space is not quite Calabi-Yau, but in some sense, close. As shown in [22], this space has 2-torsion in $H^{2}\left(X, \mathcal{O}_{X}^{*}\right)$.

\section{Parallel coincident branes on $S \hookrightarrow X$}

Let $i: S \hookrightarrow X$ be a smooth complex submanifold of a Calabi-Yau $X$, and let $\omega \in H^{2}\left(X, \mathcal{O}_{X}^{*}\right)$ define a flat $B$ field. Let $\mathcal{E}, \mathcal{F}$ be two $i^{*} \omega$-twisted holomorphic bundles on $S$, corresponding to the twisted bundles on the worldvolumes of two sets of branes, both wrapped on $S$.

Since we are assuming the $B$ field is flat, locally we can gauge the $B$ field to zero, so the physics analysis proceeds exactly as in [5], except that the Chan-Paton factors now couple to twisted bundles rather than honest bundles [14]. We shall omit the detailed explanation (consult [5] for details). The boundary Ramond sector states are of the form

$$
b_{\bar{\imath}_{1} \cdots \bar{\tau}_{n}}^{\alpha \beta j_{1} \cdots j_{m}}\left(\phi_{0}\right) \eta^{\bar{\tau}_{1}} \cdots \eta^{\bar{\tau}_{n}} \theta_{j_{1}} \cdots \theta_{j_{m}}
$$

where

$$
\begin{aligned}
\eta^{\bar{\imath}} & =\psi_{+}^{\bar{\imath}}+\psi_{-}^{\bar{\imath}} \\
\theta_{i} & =g_{i \bar{\jmath}}\left(\psi_{+}^{\bar{\jmath}}-\psi_{-}^{\bar{\jmath}}\right)
\end{aligned}
$$

as BRST invariants in the standard notation for the $B$ model [23], subject to the boundary conditions $\eta^{\overline{ }}=0$ for Dirichlet directions, and naively $\theta_{i}=0$ for Neumann directions. (The boundary conditions are modified by the ChanPaton factors, as in $[24,25]$, a point we shall return to momentarily.) The indices $\alpha, \beta$ are Chan-Paton indices, describing the $i^{*} \omega$-twisted holomorphic bundles $\mathcal{E}, \mathcal{F}$ on $S$.

Naively, physical states of the form above should be in one-to-one correspondence with elements of the sheaf cohomology groups $H^{n}\left(S, \mathcal{E}^{\vee} \otimes \mathcal{F} \otimes\right.$ 
$\left.\Lambda^{m} \mathcal{N}_{S / X}\right)$

Note that although $\mathcal{E}$ and $\mathcal{F}$ are both twisted bundles, instead of honest bundles, we can still speak of ordinary bundle-valued sheaf cohomology, since the product $\mathcal{E}^{\vee} \otimes \mathcal{F}$ is untwisted.

As in [5], there is a spectral sequence

$$
H^{p}\left(S, \mathcal{E}^{\vee} \otimes \mathcal{F} \otimes \Lambda^{q} \mathcal{N}_{S / X}\right) \Longrightarrow \operatorname{Ext}_{X, \omega}^{p+q}\left(i_{*} \mathcal{E}, i_{*} \mathcal{F}\right)
$$

relating the sheaf cohomology above to the desired ( $\omega$-twisted) Ext groups. (See appendix A for proofs.)

How is this spectral sequence realized physically? As in [5], there are two important subtleties that must be taken into account:

1. $\left.T X\right|_{S}$ need not split holomorphically, i.e. $\left.T X\right|_{S} \not T S \oplus \mathcal{N}_{S / X}$ in general, so it is not quite right to interpret the vertex operators as $\mathcal{N}_{S / X}$-valued forms.

2. The boundary conditions are twisted, by the curvature of the ChanPaton factors and the $B$ field (see [24], [25, (3.3)]), so that for Neumann directions, $\theta_{i} \neq 0$, but rather $\theta_{i}=\left(\operatorname{Tr} F_{i \bar{\jmath}}+B_{i \bar{\jmath}}\right) \eta^{\bar{\jmath}}$.

Just as in [5], in the special case that $\mathcal{E}=\mathcal{F}$, we shall see explicitly that taking care of these two subtleties has the effect of physically realizing the spectral sequence in BRST cohomology. Furthermore, we conjecture that the same result is true in general: being careful about these subtleties should have the effect of realizing all spectral sequences we discuss in this paper directly in BRST cohomology, so that the physical massless boundary Ramond sector states are in one-to-one correspondence with elements of Ext groups.

Let us specialize to the case $\mathcal{E}=\mathcal{F}$, to see how the spectral sequence is realized explicitly. As the argument is essentially identical to that in [5], we shall be brief. We deal with both of the subtleties above in the same form as in [5]. The first subtlety is dealt with by lifting $\mathcal{N}_{S / X}$-valued forms to $\left.T X\right|_{S}$-valued forms, which can be directly associated to vertex operators, unlike $\mathcal{N}_{S / X}$-valued forms. The resulting $\left.T X\right|_{S^{-}}$-valued forms need not be holomorphic, however. Their images under $\bar{\partial}$ are $T S$-valued forms, to which we can apply the boundary condition $\theta_{i}=\left(\operatorname{Tr} F_{i \bar{\jmath}}+B_{i \bar{\jmath}}\right) \eta^{\bar{\jmath}}$. Thus, the BRST operator acting on the $\left.T X\right|_{S^{-}}$valued forms can be interpreted as a composition of $\bar{\partial}$ and an evaluation map. For example, starting from an 
element of $H^{0}\left(S, \mathcal{E}^{\vee} \otimes \mathcal{E} \otimes \mathcal{N}_{S / X}\right)$, we have the composition

$$
H^{0}\left(S, \mathcal{E}^{\vee} \otimes \mathcal{E} \otimes \mathcal{N}_{S / X}\right) \stackrel{\delta}{\longrightarrow} H^{1}\left(S, \mathcal{E}^{\vee} \otimes \mathcal{E} \otimes T S\right) \stackrel{\text { eval }}{\longrightarrow} H^{2}\left(S, \mathcal{E}^{\vee} \otimes \mathcal{E}\right)
$$

where the first map $(\delta)$ is the coboundary map, and the second map (eval) is the evaluation map realizing the boundary conditions. This argument is identical to that used in [5], except for the fact that here $\mathcal{E}$ is an $i^{*} \omega$-twisted holomorphic bundle, not an honest holomorphic bundle. Also just as in [5], the composition above is exactly the differential $d_{2}$ of the spectral sequence.

Finally, as in $[6]$, we still have an Atiyah class $a(\mathcal{E}) \in H^{1}\left(S, \Omega^{1} \otimes \operatorname{End}(\mathcal{E})\right)$ corresponding to the canonical extension

$$
0 \rightarrow \Omega^{1} \otimes \mathcal{E} \rightarrow j(\mathcal{E}) \rightarrow \mathcal{E} \rightarrow 0
$$

of twisted sheaves defined by the twisted sheaf of 1 -jets of $\mathcal{E}$. The second map is simply cup product with $a(\mathcal{E})$. Note that $\Omega^{1} \otimes \operatorname{End}(\mathcal{E})$ is an ordinary sheaf. The computation proceeds exactly as in $[5,6]$. The only change is that the boundary conditions get modified as we have discussed above, corresponding to (3) being an extension of twisted sheaves rather than of ordinary sheaves.

Thus, we have seen explicitly that in the special case $\mathcal{E}=\mathcal{F}$, the spectral sequence is realized directly in BRST cohomology. We conjecture that the same statement is true for more general $\mathcal{E}, \mathcal{F}$.

\section{Example}

In this section we shall briefly consider an example involving branes wrapped on a submanifold of a space with nontrivial torsion in $H^{2}\left(X, \mathcal{O}_{X}^{*}\right)$.

Now, as mentioned earlier, we do not have any examples of Calabi-Yau's with such a property. However, we do have some examples of spaces that are not quite Calabi-Yau, and for the purposes of illustrating how to perform computations using the technology presented here, such an example will do. Of course, since the space $X$ is not Calabi-Yau, our computations will be purely formal in nature - they cannot reflect any physics, since the B model cannot be defined on $X$.

Recall earlier we mentioned an example taken from the appendix of [22]. Let $W \subset \mathbf{P}^{3} \times \mathbf{P}^{3}$ be a generic hypersurface of bidegree $(2,2)$, and let $K \subset \mathbf{P}^{3}$ be the discriminant locus of the fibration $p_{1}: W \rightarrow \mathbf{P}^{3}$, where $p_{1}$ is the projection onto the first factor. One can show that $K$ is an octic surface 
with 80 ordinary nodes. Let $d: X \rightarrow \mathbf{P}^{3}$ be the double cover of $\mathbf{P}^{3}$ branched over $K$, and let $\pi: \tilde{X} \rightarrow X$ be the blowup of the 80 nodes of $X$, so that $\tilde{X}$ is smooth.

The discussion in [22] identifies a 2-torsion element $\alpha \in H^{2}\left(\tilde{X}, \mathcal{O}_{\tilde{X}}^{*}\right)$ by exhibiting a $\mathbf{P}^{1}$ bundle over $X$ minus its nodes, and showing that this is not the projectivization of a vector bundle $\mathcal{E}$. After blowing up the nodes, this argument can be transferred to $\tilde{X}$.

In other words, the $\mathbf{P}^{1}$ bundle of [22] is the projectivization of an $\alpha$ twisted bundle $\mathcal{E}$ on $\tilde{X}$.

Let us imagine (formally) working with the B model on $\tilde{X}$, and wrapping D-branes on all of $\tilde{X}$ with twisted bundle $\mathcal{E}$. Since we are wrapping all of $\tilde{X}$, the spectral sequence is unnecessary. In any case, the spectral sequence degenerates and $\operatorname{Ext}_{\tilde{X}, \omega}^{i}(\mathcal{E}, \mathcal{E}) \simeq H^{i}\left(\mathcal{E}^{*} \otimes \mathcal{E}\right)$. Note that $\mathcal{E}^{*} \otimes \mathcal{E}$ is an ordinary bundle (the associated Azumaya algebra of Brauer group theory). In passing, note that it is only because the $B$ field is nontrivial that we could consider such twisted bundles on the brane worldvolume - were the $B$ field to be zero, for example, no such branes would exist.

Now let $D_{i}$ be the exceptional divisor of the blowup of one of the 80 nodes. Each $D_{i} \simeq \mathbf{P}^{1} \times \mathbf{P}^{1}$. In particular, there is no torsion in $H^{2}\left(D_{i}, \mathcal{O}_{D_{i}}^{*}\right)$ or $H^{3}\left(D_{i}, \mathbf{Z}\right)$ and so the $B$ field can be trivialized after restricting to $D_{i}$. Thus the gauge fields on $D$-branes wrapping $D_{i}$ live in ordinary bundles.

If $i$ and $j$ are distinct, they are disjoint and so

$$
\operatorname{Ext}^{i}\left(i_{*} \mathcal{O}_{D_{i}}, j_{*} \mathcal{O}_{D_{j}}\right)=0
$$

where $i: D_{i} \hookrightarrow \tilde{X}$ and $j: D_{j} \hookrightarrow \tilde{X}$ are the natural inclusions.

Furthermore, the spectral sequence for

$$
\operatorname{Ext}^{i}\left(i_{*} \mathcal{O}_{D_{i}}, i_{*} \mathcal{O}_{D_{i}}\right)
$$

is identical to the corresponding spectral sequence in the absence of a $B$-field, so the presence of the $B$-field does not affect the spectrum.

\section{Parallel branes on submanifolds of different di- mension}

Let $\omega \in H^{2}\left(X, \mathcal{O}_{X}^{*}\right)$ define the $B$ field, let $i: S \hookrightarrow X$ and $j: T \hookrightarrow X$ be smooth complex submanifolds of $X$ with $T \subseteq S$, and let $\mathcal{E}, \mathcal{F}$ be $i^{*} \omega$-twisted 
holomorphic bundles on $S, T$, respectively, corresponding to branes wrapped on $S$ and $T$.

The physical analysis in this case proceeds just as in [5]. The massless boundary Ramond sector states are of the form

$$
b_{\bar{\imath}_{1} \cdots \bar{\imath}_{n}}^{\alpha \beta j_{1} \cdots j_{m}}\left(\phi_{0}\right) \eta^{\bar{\imath}_{1}} \cdots \eta^{\bar{\imath}_{n}} \theta_{j_{1}} \cdots \theta_{j_{m}}
$$

where $\alpha, \beta$ are Chan-Paton factors describing the $j^{*} \omega$-twisted bundles $\left.\mathcal{E}\right|_{T}$ and $\mathcal{F}$, the $\eta$ indices are tangent to $T$, and (momentarily ignoring the twisting of $[24,25])$ the $\theta$ indices are normal to $S \hookrightarrow X$. Naively, the BRST cohomology classes of such states appear to be counted by the sheaf cohomology groups $H^{n}\left(T,\left.\left.\mathcal{E}^{\vee}\right|_{T} \otimes \mathcal{F} \otimes \Lambda^{m} \mathcal{N}_{S / X}\right|_{T}\right)$.

Exactly as in [5], there is a spectral sequence

$$
H^{n}\left(T,\left(\left.\mathcal{E}\right|_{T}\right)^{\vee} \otimes \mathcal{F} \otimes \Lambda^{m} \mathcal{N}_{S / X}\right) \Longrightarrow \operatorname{Ext}_{X, \omega}^{n+m}\left(i_{*} \mathcal{E}, j_{*} \mathcal{F}\right)
$$

(See appendix A for proofs.)

Exactly as in [5], we have neglected certain subtleties, described in more detail in the previous section, and we conjecture that when those subtleties are taken into account, we are left with BRST cohomology classes counted by the $\omega$-twisted Ext groups above, as we explicitly saw happens for parallel coincident branes in the previous section.

\section{$6 \quad$ General intersecting branes}

The analysis for general intersections proceeds much as in the above and in [5]. Let $S, T$ be smooth submanifolds, and let $\mathcal{E}, \mathcal{F}$ be twisted holomorphic vector bundles on $S, T$, respectively, each twisted by the pullback of an element of $H^{2}\left(X, \mathcal{O}_{X}^{*}\right)$. In order to write down the massless boundary Ramond sector states for the general case, we need to take into account facts about branes at angles [26]. We refer the reader to [5] for details of the analysis in the untwisted case, and the analysis in the twisted case is a nearly exact duplicate. Suffice it to say that the massless boundary Ramond sector spectrum, ignoring subtleties in boundary conditions, appears to be counted by the sheaf cohomology groups

$$
\begin{aligned}
& H^{p}\left(S \cap T,\left.\left.\mathcal{E}^{\vee}\right|_{S \cap T} \otimes \mathcal{F}\right|_{S \cap T} \otimes \Lambda^{q-m} \tilde{N} \otimes \Lambda^{t o p} \mathcal{N}_{S \cap T / T}\right) \\
& H^{p}\left(S \cap T,\left.\left.\mathcal{E}\right|_{S \cap T} \otimes \mathcal{F}^{\vee}\right|_{S \cap T} \otimes \Lambda^{q-n} \tilde{N} \otimes \Lambda^{t o p} \mathcal{N}_{S \cap T / S}\right)
\end{aligned}
$$

where

$$
\tilde{N}=\left.T X\right|_{S \cap T} /\left(\left.T S\right|_{S \cap T}+\left.T T\right|_{S \cap T}\right)
$$


and $m=\operatorname{rk} \mathcal{N}_{S \cap T / T}, n=\operatorname{rk} \mathcal{N}_{S \cap T / S}$.

Just as in [5], we are led to believe the boundary Fock vacua are sections of the line bundles $\Lambda^{t o p} \mathcal{N}_{S \cap T / T}$ and $\Lambda^{t o p} \mathcal{N}_{S \cap T / S}$, in order to get any relationship to Ext groups. As before, this statement is supported by analysis of anomalies, and suggests implications for supersymmetry breaking, and is also required to restore Serre duality invariance of the spectrum. These subtleties are all discussed in great detail in [5], and we refer the reader there for more information.

Exactly as in [5], there are two spectral sequences

$$
\begin{aligned}
H^{p}\left(S \cap T,\left.\left.\mathcal{E}^{\vee}\right|_{S \cap T} \otimes \mathcal{F}\right|_{S \cap T} \otimes \Lambda^{q-m} \tilde{N} \otimes \Lambda^{t o p} \mathcal{N}_{S \cap T / T}\right) & \Longrightarrow \operatorname{Ext}_{X, \omega}^{p+q}\left(i_{*} \mathcal{E}, j_{*} \mathcal{F}\right) \\
H^{p}\left(S \cap T,\left.\left.\mathcal{E}\right|_{S \cap T} \otimes \mathcal{F}^{\vee}\right|_{S \cap T} \otimes \Lambda^{q-n} \tilde{N} \otimes \Lambda^{t o p} \mathcal{N}_{S \cap T / S}\right) & \Longrightarrow \operatorname{Ext}_{X, \omega}^{p+q}\left(j_{*} \mathcal{F}, i_{*} \mathcal{E}\right)
\end{aligned}
$$

(See appendix A for proofs.)

All the subtleties that arose in [5] have immediate analogues here. For purposes of brevity, we merely refer the reader to [5] for more details. Just as in [5], we conjecture that both of the spectral sequences above are realized physically in BRST cohomology, due ultimately to Chan-Paton-induced boundary condition twistings of the form described in [24, 25], as we explicitly saw happens earlier in the special case of parallel coincident branes.

\section{Conclusions}

In this paper we have explicitly computed the massless boundary Ramond sector spectrum in open strings between D-branes wrapped on holomorphic submanifolds, with nontrivial flat $B$ fields turned on, carrying on the program of $[5,6]$. Just as in our earlier work $[5,6]$, we find that the spectra are again counted by Ext groups, this time Ext groups between twisted sheaves, where the twist is determined by the $B$ field.

It has been previously proposed $[2,3,4]$ that some properties of D-branes could be modeled by derived categories. The proposals in $[2,3,4]$, however, really only speak to a special class of D-branes, namely, those with no background fields turned on - no $B$ field, and no Higgs field vevs. In this paper we begin to see how some of those constraints can be lifted. In the presence of a nontrivial flat $B$ field, for example, we have given concrete evidence that ordinary derived categories should be replaced by derived categories of twisted sheaves. (See also [16] for some previous work in this vein.) It should 
also be possible to lift some of the other restrictions mentioned. For example, it has been suggested [27] that giving nilpotent vevs to Higgs fields might be modeled sheaf-theoretically in terms of sheaves on nonreduced subschemes of Calabi-Yau's. Work to compare open string spectra in such backgrounds with Ext groups between sheaves on nonreduced subschemes is in progress.

\section{Acknowledgments}

We would like to thank D. Morrison for useful conversations. The work of A.C. is partially supported by an NSF Postdoctoral Research Fellowship. The work of S.K. is partially supported by NSF grant DMS 02-96154 and NSA grant MDA904-02-1-0024. The work of E.S. is partially supported by NSF grant DMS 02-96154.

\section{A Proofs of spectral sequences}

In this appendix we derive the spectral sequences used in the rest of the paper. We work with a background smooth variety (or complex manifold) $X$, endowed with a $B$-field $\alpha \in \operatorname{Br}(X)$. We consider branes $(S, \mathcal{E})$ and $(T, \mathcal{F})$ supported at the smooth subvarieties $S$ and $T$ of $X$. We let $W$ be their scheme theoretic intersection, and we label the inclusions as follows:

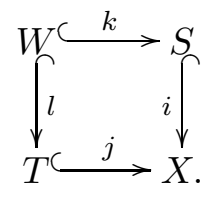

The bundles $\mathcal{E}$ and $\mathcal{F}$ are twisted by $i^{*} \alpha$ and $j^{*} \alpha$ on $S$ and $T$, respectively. We assume that $W$ is smooth; in concrete terms this means that the set theoretic intersection of $S$ and $T$ is smooth, and furthermore the equality

$$
T_{w, W}=T_{w, S} \cap T_{w, T}
$$

holds at every point $w \in W$.

The main spectral sequence that we want to derive is:

Theorem A.1. Let $m$ be the rank of $N_{W / T}$, the normal bundle of $W$ in $T$, and let $\tilde{N}$ be the vector bundle on $W$ given by

$$
\tilde{N}=\left.T_{X}\right|_{W} /\left(\left.T_{S}\right|_{W}+\left.T_{T}\right|_{W}\right) .
$$


There exists a convergent spectral sequence

$$
{ }^{2} E^{p q}=H^{p}\left(W,\left.\left.\mathcal{E}^{\vee}\right|_{W} \otimes \mathcal{F}\right|_{W} \otimes \Lambda^{m} \mathcal{N}_{W / T} \otimes \Lambda^{q-m} \tilde{N}\right) \Longrightarrow \operatorname{Ext}_{X}^{p+q}\left(i_{*} \mathcal{E}, j_{*} \mathcal{F}\right) .
$$

We use the convention that $\Lambda^{0} \tilde{N}=\mathcal{O}_{W}$ for a bundle $\tilde{N}$ on $W$, even if $\tilde{N}=0$. $\mathcal{E}^{\vee}$ denotes the dual of the bundle $\mathcal{E}$.

Since $\mathcal{E}$ and $\mathcal{F}$ are twisted by $i^{*} \alpha$ and $j^{*} \alpha$, respectively, $i_{*} \mathcal{E}$ and $j_{*} \mathcal{F}$ are defined, and they are both $\alpha$-twisted, $[13,1.2 .10]$. Thus $\underline{\operatorname{Ext}}_{X}^{p+q}\left(i_{*} \mathcal{E}, j_{*} \mathcal{F}\right)$ is untwisted and hence $\operatorname{Ext}_{X}^{p+q}\left(i_{*} \mathcal{E}, j_{*} \mathcal{F}\right)$ makes sense. See [13, 1.2.12] for details. Similarly, $\left.\left.\mathcal{E}^{\vee}\right|_{W} \otimes \mathcal{F}\right|_{W}$ is untwisted, so the ${ }^{2} E^{p q}$ term is defined.

Before we can proceed to the proof of Theorem A.1 we need several auxiliary results.

Proposition A.2. Around every point $w \in W$ there exists an open neighborhood $U \subseteq X$ and a smooth subvariety $Y$ of $U$ containing $w$ such that

a) $S \subseteq Y$,

b) $W \cap U$ is the scheme theoretic intersection of $Y$ and $T$, and

c) $Y$ and $T$ intersect in the expected dimension, i.e.

$$
\operatorname{dim} Y+\operatorname{dim} T-\operatorname{dim} X=\operatorname{dim} W .
$$

Proof. Proceed by induction on $n=\operatorname{dim} T-\operatorname{dim} W$. If $n=0$, then $T \subseteq S$ and we can take $Y=X$. If $n>0$, then $\operatorname{dim} W<\operatorname{dim} T$, and since we assumed that

$$
T_{W, w}=T_{T, w} \cap T_{S, w},
$$

we conclude that $T_{T, w}$ is not contained in $T_{S, w}$.

Observe that $T_{S, w}$ is the intersection of the (Zariski) tangent spaces $T_{X^{\prime}, w}$ of all the local hypersurfaces $X^{\prime}$ through $w$ that contain $S$. Since $T_{T, w}$ is not contained in $T_{S, w}$, there exists a hypersurface $X^{\prime}$ that contains $S$ and such that $T_{T, w} \nsubseteq T_{X^{\prime}, w}$. Thus $X^{\prime}$ and $T^{\prime}=T \cap X$ are smooth at $w$, of dimensions $\operatorname{dim} X-1$ and $\operatorname{dim} T-1$, respectively, and $S \subseteq X^{\prime}$. We have

$$
S \cap T^{\prime}=S \cap\left(X^{\prime} \cap T\right)=\left(S \cap X^{\prime}\right) \cap T=S \cap T=W,
$$

thus we are in the same situation as before, but with $\operatorname{dim} T^{\prime}-\operatorname{dim} W=n-1$. By the induction hypothesis we can find $Y$ in $X^{\prime}$ satisfying the conditions 
of the proposition. Considering $Y$ inside of $X$ instead of $X^{\prime}$ still satisfies (a) and (b), and we have

$$
\begin{aligned}
\operatorname{dim} Y+\operatorname{dim} T-\operatorname{dim} X & =\operatorname{dim} Y+\left(\operatorname{dim} T^{\prime}+1\right)-\left(\operatorname{dim} X^{\prime}+1\right) \\
& =\operatorname{dim} Y+\operatorname{dim} T^{\prime}-\operatorname{dim} X^{\prime}=\operatorname{dim} W .
\end{aligned}
$$

Proposition A.3. If $S \subseteq T$ then

$$
\operatorname{Tor}_{q}^{X}\left(\mathcal{O}_{S}, \mathcal{O}_{T}\right)=\left.\Lambda^{q} \mathcal{N}_{T / X}^{\vee}\right|_{S},
$$

where $\mathcal{N}_{T / X}$ is the normal bundle of $T$ in $X$. (We abuse notation and write $\mathcal{O}_{S}$ for $i_{*} \mathcal{O}_{S}$, etc.)

Proof. Since the question is local and $T$ is locally a complete intersection, we can use the Koszul resolution with respect to the normal bundle to obtain a locally free resolution of $\mathcal{O}_{T}$

$$
\cdots \rightarrow \Lambda^{q+1} \mathcal{G}^{\vee} \rightarrow \Lambda^{q} \mathcal{G}^{\vee} \rightarrow \cdots \rightarrow \mathcal{G}^{\vee} \rightarrow \mathcal{O}_{X} \rightarrow \mathcal{O}_{T} \rightarrow 0
$$

around every point of $T$, where $\mathcal{G}$ is locally free on $X$ and $\left.\mathcal{G}\right|_{T} \simeq \mathcal{N}_{T / X}$. To compute $\operatorname{Tor}_{q}^{X}\left(\mathcal{O}_{S}, \mathcal{O}_{T}\right)$ we tensor this resolution by $\mathcal{O}_{S}$, which has the effect of restricting all the bundles to $S$ and making all the maps in the complex zero, hence the result.

Proposition A.4 ( [28, Corollary to Théorème 4, p. V-20]). If $W$ is of the expected dimension, i.e.,

$$
\operatorname{dim} S+\operatorname{dim} T-\operatorname{dim} X=\operatorname{dim} W
$$

then

$$
\operatorname{Tor}_{q}^{X}\left(\mathcal{O}_{S}, \mathcal{O}_{T}\right)=0
$$

for $q>0$.

\section{Proposition A.5.}

$$
\operatorname{Tor}_{q}^{X}\left(\mathcal{O}_{S}, \mathcal{O}_{T}\right)=\Lambda^{q} \tilde{N}^{\vee}
$$

where $\tilde{N}=T_{X} /\left(T_{S}+T_{T}\right)$.

Proof. By Proposition A.2, around every point of $W$ we can find $Y$ smooth such that $S$ is contained in $Y, T$ and $Y$ intersect in the expected dimension, and $W=T \cap Y$. 
Since $T$ and $Y$ intersect in the expected dimension, using Proposition A.4 we see that the change-of-rings spectral sequence [29, Exercise A3.45]

$$
\operatorname{Tor}_{q}^{Y}\left(\mathcal{O}_{S}, \operatorname{Tor}_{p}^{X}\left(\mathcal{O}_{T}, \mathcal{O}_{Y}\right)\right) \Longrightarrow \operatorname{Tor}_{p+q}^{X}\left(\mathcal{O}_{S}, \mathcal{O}_{T}\right)
$$

consists of the single column

$$
\operatorname{Tor}_{q}^{Y}\left(\mathcal{O}_{S}, \operatorname{Tor}_{0}^{X}\left(\mathcal{O}_{T}, \mathcal{O}_{Y}\right)\right)=\operatorname{Tor}_{q}^{Y}\left(\mathcal{O}_{S}, \mathcal{O}_{W}\right)=\left.\Lambda^{q} \mathcal{N}_{S / Y}^{\vee}\right|_{W}
$$

where the last equality is Proposition A.3. In other words,

$$
\operatorname{Tor}_{q}^{X}\left(\mathcal{O}_{S}, \mathcal{O}_{T}\right)=\left.\Lambda^{q} \mathcal{N}_{S / Y}^{\vee}\right|_{W}
$$

At each $w \in W$, the composition of maps

$$
\mathcal{N}_{S / Y, w}=T_{Y, w} / T_{S, w} \rightarrow T_{X, w} / T_{S, w} \rightarrow T_{X, w} /\left(T_{S, w}+T_{T, w}\right)=\tilde{N}_{w}
$$

is injective, because a tangent vector to $Y$ will be tangent to $T$ only if it is tangent to $W$, and then it is tangent to $S$. Comparing dimensions, we see that the natural map $\mathcal{N}_{S / Y} \rightarrow \tilde{N}$ is therefore an isomorphism. Finally, one can trace through the identifications and see that the local isomorphism

$$
\operatorname{Tor}_{q}^{X}\left(\mathcal{O}_{S}, \mathcal{O}_{T}\right) \cong \Lambda^{q} \tilde{N}^{\vee}
$$

does not depend on the choice of $Y$, hence it lifts to a global isomorphism.

As this calculation can be tedious, we supplement this argument with an alternative approach. The claimed result is elementary to check directly for $q=0$. For $q=1$ this is also straightforward: using the exact sequence

$$
0 \rightarrow \mathcal{I}_{S} \rightarrow \mathcal{O}_{X} \rightarrow \mathcal{O}_{S} \rightarrow 0,
$$

we compute that $\operatorname{Tor}_{1}^{X}\left(\mathcal{O}_{S}, \mathcal{O}_{T}\right)$ is isomorphic to the kernel of the map

$$
\mathcal{I}_{S} \otimes \mathcal{O}_{T} \rightarrow \mathcal{O}_{T}
$$

namely

$$
\frac{\mathcal{I}_{S} \cap \mathcal{I}_{T}}{\mathcal{I}_{S} \mathcal{I}_{T}}
$$

But this can be seen directly to be isomorphic to $\tilde{N}^{\vee}$; the isomorphism is induced by the natural map $\left(\mathcal{I}_{S} \cap \mathcal{I}_{T}\right) /\left(\mathcal{I}_{S} \mathcal{I}_{T}\right) \rightarrow \tilde{N}^{\vee}$ taking a class represented by a function $f$ to the linear functional on $\tilde{N}$ induced by $d f$.

For $q>1$ we note the existence of a natural map

$$
\Lambda^{q} \operatorname{Tor}_{1}^{X}\left(\mathcal{O}_{S}, \mathcal{O}_{T}\right) \rightarrow \operatorname{Tor}_{q}^{X}\left(\mathcal{O}_{S}, \mathcal{O}_{T}\right)
$$


This alternating product is constructed by using the exterior algebra structure on any local Koszul resolution and the multiplication on $\mathcal{O}_{T}$. As the algebra structure on the Koszul complex is canonical (a change of regular sequence induces a canonical isomorphism of algebras), the map (4) is canonical, independent of the choice of local defining equations yielding the Koszul complex. Then our earlier local calculations show that (4) is an isomorphism.

From here on we switch to the language of derived categories. Recall ([13], [30]) that objects in the derived category of twisted coherent sheaves on a space (or abelian groups, etc.) are complexes of such sheaves, with morphisms defined using the machinery of derived categories. For a morphism between smooth spaces (as well as in much more general cases), one has derived functors corresponding to push forward, pull back, $\otimes$, Hom, $\underline{\text { Hom}}$, etc. Applying any of these functors to a complex yields again a complex. Given such a complex $\mathcal{C}$ (of twisted sheaves, abelian groups, etc.) we can consider its $q$-th cohomology object, denoted by $\mathcal{H}^{q}(\mathcal{C})$. It is a twisted sheaf or an abelian group, accordingly. We use the convention that $\mathcal{H}_{q}(\mathcal{C})=\mathcal{H}^{-q}(\mathcal{C})$

Note that pushing forward by an affine morphism is exact, so we do not need to derive this functor. Also, tensoring by a locally free sheaf is exact, and this tensoring operation does not need to be derived either. On the other hand, pulling back to a subvariety is only right exact, so we need to left-derive it to get a well-defined functor on derived categories.

Proposition A.6. We have

$$
\mathcal{H}_{q}\left(\mathbf{L} j^{*} i_{*} \mathcal{E}\right)=l_{*}\left(\left.\left.\mathcal{E}\right|_{W} \otimes \mathcal{H}_{q}\left(\mathbf{L} i^{*} j_{*} \mathcal{O}_{T}\right)\right|_{W}\right) .
$$

Combined with Proposition A.5, this yields

$$
\mathcal{H}_{q}\left(\mathbf{L} j^{*} i_{*} \mathcal{E}\right)=l_{*}\left(\left.\mathcal{E}\right|_{W} \otimes \Lambda^{q} \tilde{N}^{\vee}\right) .
$$

Proof. By the projection formula ([13, 2.3.5], [30, II.5.6]) we have

$$
j_{*} \mathbf{L} j^{*} i_{*} \mathcal{E}=i_{*} \mathcal{E} \stackrel{\mathbf{L}}{\otimes} j_{X} j_{*} \mathcal{O}_{T}
$$

On the other hand, since $j_{*}$ is exact, we have

$$
\mathcal{H}_{q}\left(j_{*} \mathbf{L} j^{*} i_{*} \mathcal{E}\right)=j_{*} \mathcal{H}_{q}\left(\mathbf{L} j^{*} i_{*} \mathcal{E}\right)
$$

and thus

$$
\mathcal{H}_{q}\left(\mathbf{L} j^{*} i_{*} \mathcal{E}\right)=j^{*} \mathcal{H}_{q}\left(j_{*} \mathbf{L} j^{*} i_{*} \mathcal{E}\right)=j^{*} \mathcal{H}_{q}\left(i_{*} \mathcal{E} \stackrel{\mathbf{L}}{\otimes_{X}} j_{*} \mathcal{O}_{T}\right)
$$


(Here we have used the fact that for a sheaf or twisted sheaf, pulling back in the underived way its push-forward by a closed embedding yields back the original sheaf.) Using the projection formula again for $i_{*}$ we have

$$
i_{*} \mathcal{E} \stackrel{\mathbf{L}}{\otimes} j_{X} j_{*} \mathcal{O}_{T}=i_{*}\left(\mathcal{E} \otimes_{S} \mathbf{L} i^{*} j_{*} \mathcal{O}_{T}\right),
$$

and since $\mathcal{E}$ is locally free on $S$ we get

$$
\mathcal{H}_{q}\left(i_{*} \mathcal{E} \stackrel{\mathbf{L}}{\otimes_{X}} j_{*} \mathcal{O}_{T}\right)=i_{*} \mathcal{H}_{q}\left(\mathcal{E} \otimes_{S} \mathbf{L} i^{*} j_{*} \mathcal{O}_{T}\right)=i_{*}\left(\mathcal{E} \otimes_{S} \mathcal{H}_{q}\left(\mathbf{L} i^{*} j_{*} \mathcal{O}_{T}\right)\right) .
$$

But for sheaves and underived operations, $j^{*} i_{*}=l_{*} k^{*}$. Therefore

$$
\begin{aligned}
\mathcal{H}_{q}\left(\mathbf{L} j^{*} i_{*} \mathcal{E}\right) & =j^{*} i_{*}\left(\mathcal{E} \otimes_{S} \mathcal{H}_{q}\left(\mathbf{L} i^{*} j_{*} \mathcal{O}_{T}\right)\right) \\
& =l_{*} k^{*}\left(\mathcal{E} \otimes_{S} \mathcal{H}_{q}\left(\mathbf{L} i^{*} j_{*} \mathcal{O}_{T}\right)\right) \\
& =l_{*}\left(\left.\left.\mathcal{E}\right|_{W} \otimes \mathcal{H}_{q}\left(\mathbf{L} i^{*} j_{*} \mathcal{O}_{T}\right)\right|_{W}\right)
\end{aligned}
$$

which is the first result. Applying again the projection formula we have

$$
\mathcal{H}_{q}\left(\mathbf{L} i^{*} j_{*} \mathcal{O}_{T}\right)=i^{*} \mathcal{H}_{q}\left(i_{*} \mathcal{O}_{S} \stackrel{\mathbf{L}}{\otimes}{ }_{X} j_{*} \mathcal{O}_{T}\right)=i^{*} \operatorname{Tor}_{q}^{X}\left(\mathcal{O}_{S}, \mathcal{O}_{T}\right)
$$

By Proposition A.5,

$$
\operatorname{Tor}_{q}^{X}\left(\mathcal{O}_{S}, \mathcal{O}_{T}\right)=\Lambda^{q} \tilde{N}^{\vee}
$$

hence the second result.

Proposition A.7. If $l: W \hookrightarrow T$ is a locally complete intersection embedding of varieties or complex analytic spaces of relative dimension $m$ (such is the case if, for example, $W$ is a smooth subvariety of the smooth variety $T$ ), and $\alpha$ is an element of $\operatorname{Br}(T)$, then we have a natural isomorphism

$$
\mathbf{R}_{\operatorname{Hom}_{T}}\left(l_{*} \mathcal{G}, \mathcal{F}\right) \cong l_{*} \underline{\operatorname{Rom}}_{W}\left(\mathcal{G}, l^{!} \mathcal{F}\right)
$$

for $\mathcal{G} \in \mathbf{D}_{\mathrm{coh}}^{b}\left(W, l^{*} \alpha\right)$ and $\mathcal{F} \in \mathbf{D}_{\mathrm{coh}}^{b}(T, \alpha)$, where

$$
l^{!}(\mathcal{F})=\mathbf{L} l^{*}(\mathcal{F}) \otimes \Lambda^{m} \mathcal{N}_{W / T}[-m]
$$

(Here, $\mathcal{N}_{W / T}=\left(\mathcal{I}_{W} / \mathcal{I}_{W}^{2}\right)^{\vee}$, and $[-m]$ denotes the shift by $-m$.)

Applying $\mathbf{R} \Gamma$ to both sides we conclude that $l^{!}$is a right adjoint to $l_{*}$, as functors between $\mathbf{D}_{\mathrm{coh}}^{b}\left(W, l^{*} \alpha\right)$ and $\mathbf{D}_{\mathrm{coh}}^{b}(T, \alpha)$.

Proof. To prove the first statement it is enough to show that there exist natural local isomorphisms

$$
\mathbf{R}_{\operatorname{Hom}_{U}}\left(\left.l_{*} \mathcal{G}\right|_{U},\left.\mathcal{F}\right|_{U}\right)=l_{*} \underline{\operatorname{Hom}}_{V}\left(\left.\mathcal{G}\right|_{V}, l^{!}\left(\left.\mathcal{F}\right|_{V}\right)\right)
$$


for $U$ an open set of $T$, sufficiently small to trivialize $\alpha$, and $V=U \cap W$. (Here we work with either the analytic or the étale topology, as the Zariski topology is not fine enough for the Brauer group to be interesting.) In this form, the above isomorphism is nothing else than duality for a finite morphism ([30, III.7.3, III.6.7 and Definition after III.1.3]; see [31] for similar statements for morphisms of analytic spaces).

The second statement follows immediately from the fact that

$$
\mathbf{R} \Gamma\left(T, \underline{\operatorname{RHom}}_{T}(\cdot, \cdot)\right)=\mathbf{R H o m}_{T}(\cdot, \cdot)
$$

for $\alpha$-twisted sheaves [13, 2.3.2].

Now we can finally prove the main result:

Proof of the main theorem. The functor $\mathbf{L} j^{*}$ is left adjoint to $j_{*}$ ([13, 2.3.9], [30, II.5.11]), so we have

$$
\operatorname{Ext}_{X}^{p+q}\left(i_{*} \mathcal{E}, j_{*} \mathcal{F}\right)=\mathcal{H}^{p+q} \mathbf{R} \operatorname{Hom}_{X}\left(i_{*} \mathcal{E}, j_{*} \mathcal{F}\right)=\mathcal{H}^{p+q} \mathbf{R} \operatorname{Hom}_{T}\left(\mathbf{L} j^{*} i_{*} \mathcal{E}, \mathcal{F}\right)
$$

Writing down the Grothendieck spectral sequence for the composition of the two functors $\mathbf{R H o m}_{T}(\cdot, \mathcal{F})$ (contravariant) and $\mathbf{L} j^{*} i_{*}(\cdot)$ (covariant) yields the convergent spectral sequence

$$
{ }^{2} E^{p q}=\mathcal{H}^{p} \mathbf{R} \operatorname{Hom}_{T}\left(\mathcal{H}_{q}\left(\mathbf{L} j^{*} i_{*} \mathcal{E}\right), \mathcal{F}\right) \Longrightarrow \mathcal{H}^{p+q} \mathbf{R} \operatorname{Hom}_{T}\left(\mathbf{L} j^{*} i_{*} \mathcal{E}, \mathcal{F}\right) .
$$

(Note the implicit change of sign in $q$, due to the fact that $\operatorname{RHom}_{T}(\cdot, \mathcal{F})$ is contravariant.) Using Proposition A.6 we get that

$$
{ }^{2} E^{p q}=\mathcal{H}^{p} \mathbf{R} \operatorname{Hom}_{T}\left(l_{*}\left(\left.\mathcal{E}\right|_{W} \otimes \Lambda^{q} \tilde{N}^{\vee}\right), \mathcal{F}\right),
$$

which by Grothendieck duality (Proposition A.7) gives

$$
{ }^{2} E^{p q}=\mathcal{H}^{p} \mathbf{R H o m}_{W}\left(\left.\mathcal{E}\right|_{W} \otimes \Lambda^{q} \tilde{N}^{\vee},\left.\mathcal{F}\right|_{W} \otimes \Lambda^{m} \mathcal{N}_{W / T}[-m]\right) .
$$

But $\mathcal{E}$ and $\Lambda^{q} \tilde{N}^{\vee}$ are locally free, so

$$
\begin{aligned}
{ }^{2} E^{p q} & =\mathcal{H}^{p} \mathbf{R H o m}_{W}\left(\left.\mathcal{E}\right|_{W} \otimes \Lambda^{q} \tilde{N}^{\vee},\left.\mathcal{F}\right|_{W} \otimes \Lambda^{m} \mathcal{N}_{W / T}[-m]\right) \\
& =H^{p-m}\left(W,\left.\left.\mathcal{E}^{\vee}\right|_{W} \otimes \mathcal{F}\right|_{W} \otimes \Lambda^{t o p} \mathcal{N}_{W / T} \otimes \Lambda^{q} \tilde{N}\right) .
\end{aligned}
$$

Renumbering and putting it all together we arrive at our result: there exists a convergent spectral sequence

$$
{ }^{2} E^{p q}=H^{p}\left(W,\left.\left.\mathcal{E}^{\vee}\right|_{W} \otimes \mathcal{F}\right|_{W} \otimes \Lambda^{m} \mathcal{N}_{W / T} \otimes \Lambda^{q-m} \tilde{N}\right) \Longrightarrow \operatorname{Ext}_{X}^{p+q}\left(i_{*} \mathcal{E}, j_{*} \mathcal{F}\right) .
$$




\section{References}

[1] P. Aspinwall and R. Donagi, "The heterotic string, the tangent bundle, and derived categories," Adv. Theor. Math. Phys. 2 (1998) 1041-1074, hep-th/9806094.

[2] E. Sharpe, "D-branes, derived categories, and Grothendieck groups," Nucl. Phys. B561 (1999) 433-450, hep-th/9902116.

[3] M. Douglas, "D-branes, categories, and $\mathcal{N}=1$ supersymmetry," J. Math. Phys. 42 (2001) 2818-2843, hep-th/0011017.

[4] P. Aspinwall and A. Lawrence, "Derived categories and zero-brane stability," JHEP 0108 (2001) 004, hep-th/0104147.

[5] S. Katz and E. Sharpe, "D-branes, open string vertex operators, and Ext groups," hep-th/0208104.

[6] S. Katz, T. Pantev, and E. Sharpe, "D-branes, orbifolds, and Ext groups," hep-th/0212218.

[7] N. Hitchin, "Lectures on special Lagrangian submanifolds," math.DG/9907034.

[8] E. Sharpe, "Discrete torsion," hep-th/0008154.

[9] E. Sharpe, "Recent developments in discrete torsion," Phys. Lett. B498 (2001) 104-110, hep-th/0008191.

[10] M. Douglas, "D-branes and discrete torsion," hep-th/9807235.

[11] A. Kapustin, "D-branes in a topologically nontrivial $B$-field," hep-th/9909089.

[12] P. Bouwknegt and V. Mathai, "D-branes, $B$ fields, and twisted Ktheory," JHEP 0003 (2000) 007, hep-th/0002023.

[13] A. Căldăraru, "Derived categories of twisted sheaves on CalabiYau manifolds," Cornell University Ph.D. thesis, 2000. Available at http://www.math. upenn.edu/ andreic

[14] D. Freed and E. Witten, "Anomalies in string theory with D-branes," hep-th/9907189.

[15] D. Eisenbud and J. Harris, The Geometry of Schemes, Springer-Verlag, New York, 2000. 
[16] A. Kapustin and D. Orlov, "Vertex algebras, mirror symmetry, and D-branes: the case of complex tori," hep-th/0010293.

[17] C.H. Clemens and P.A. Griffiths, "The intermediate Jacobian of the cubic threefold," Ann. Math. 95 (1972), 281-356.

[18] P. Candelas, X. de la Ossa, and F. Rodriguez-Villegas, "Calabi-Yau manifolds over finite fields, I," hep-th/0012233.

[19] A. Mustaţă, Ph.D. thesis, University of Utah, 2003.

[20] C. Klimcik and P. Severa, "Open strings and D-branes in WZNW models," Nucl. Phys. B488 (1997) 653-676, hep-th/9609112.

[21] A. Alekseev and V. Schomerus, "D-branes in the WZW model," Phys. Rev. D60 (1999) 061901, hep-th/9812193.

[22] P. Aspinwall, D. Morrison, and M. Gross, "Stable singularities in string theory," Comm. Math. Phys. 178 (1996) 115-134, hep-th/9503208.

[23] E. Witten, "Mirror manifolds and topological field theory," in Mirror Symmetry I, ed. S.-T. Yau, American Mathematical Society, 1998.

[24] A. Abouelsaood, C. Callan, C. Nappi, and S. Yost, "Open strings in background gauge fields," Nucl. Phys. B280 (1987) 599-624.

[25] C. Callan, C. Lovelace, C. Nappi, and S. Yost, "String loop corrections to beta functions," Nucl. Phys. B288 (1987) 525-550.

[26] M. Berkooz, M. Douglas, and R. Leigh, "Branes intersecting at angles," Nucl. Phys. B480 (1996) 265-278, hep-th/9606139.

[27] T. Gomez and E. Sharpe, "D-branes and scheme theory," hep-th/0008150.

[28] J.-P. Serre, Algèbre Locale - Multiplicités, LNM 11, Springer-Verlag, 1965.

[29] D. Eisenbud, Commutative algebra with a view toward algebraic geometry, LNM 150, Springer-Verlag, 1995.

[30] R. Hartshorne, Residues and Duality, LNM 20, Springer-Verlag, 1966.

[31] C. Bănică and O. Stănăşilă, Algebraic methods in the global theory of complex spaces, John Wiley, New York, 1976. 